Article

\title{
Recursive Abduction and the Universality of Physical Laws: A Logical Analysis Based on Case Studies
}

\author{
Yuqing He \\ Independent researcher, Guangzhou City, Guangdong Province, China; E-mail: hyq5.27@163.com
}

\begin{abstract}
The paper studies some cases in physics such as Galilean inertia motion and etc., and presents a logical schema of recursive abduction, from which we can derive the universality of physical laws in an effective logical path without requiring infinite inductions. Recursive abduction provides an effective logical framework to connect a universal physical law with finite empirical observations based on both quasi-law tautologies and suitable recursive dimensions, two new concepts introduced in this paper. Under the viewpoint of recursive abduction, the historical difficulty from Hume's problem naturally vanishes. In Hume's problem one always misunderstood a time-recursive issue as an infinitely inductive problem and, thus, sank into an inescapable quagmire. With this new effective logical schema, the paper gives a concluding discussion to Hume's problem and justifies the validity of probability argument for natural laws.
\end{abstract}

Key words: Abduction; Recursion; Physical law; Hume's problem

MSC classifications: 00A79; 03A05; 03B60; 03C57

\section{Introduction}

The paper clarifies the properties of physical law from three aspects: abduction, the foundation of the universality of physical laws, and Hume's problem.

Abduction had revealed an intuitively vivid logical process in empirical judgments, but left the logical uncertainty with physical laws just as with commonsense. It significantly conflicts with our general impression that the physical law is much more successful than commonsense. From the initial illustration of abduction, by Pierce [1], to the more rigorous formal formulations of abduction, e.g., by Meheus and Batens [2], Lycke [3], Soler-Toscano, et al. [4], Beirlaen and Aliseda [5], Nubiola [6], and so on, during one and half centuries, physical law and commonsense were always indiscriminately viewed as similar objects in abductive analyses. Below, we shall call this kind of abduction as normal abduction, which always mixes strict natural laws with commonsense. In the framework of normal abduction, the traditional interest of abductive studies was usually not to clarify the difference between the discovery processes of physical laws and other objects, in contrast, it usually focuses on their similarity, e.g., see Pombo and Gerner [7], McNally, et al. [8], Chattopadhyay and Lipson [9], Haig [10], Khemlani, et al. [11], Pedemonte and Reid [12], Bajc [13], Singer [14], and so on, in which the interested logical inferential processes in physics, biology, psychology, pedagogy, ethnography, jurisprudence, and etc. were almost the same.

Developing from Pierce's initial proposal [1], abduction as the non-standard logic follows the reasoning schema 


$$
B(x),(A(x) \rightarrow B(x)) / A(x),
$$

where $A$ and $B$ must be different predicates. This characteristic distinguishes abduction from empirical induction. Empirical induction is always made with different quantifiers but with identical predicates. In such a characteristic framework for identifying the abductive reasoning, a physical law and commonsense are usually the same. Another traditional emphasis to abductive reasoning is its distinction from deductive reasoning, in which an abduced object is certainly inderivable in any classical logic procedure. These two traditional emphases limited researchers to use abduction in the deep analysis of physical laws.

Focusing on its distinction from induction and deduction, normal abduction deals with all abduced objects with postulations as the following, e.g., see Nubiola [6]:

i) they are not the results derived from any deductive procedure;

ii) they are purely empirical guesses.

Namely, any abduced object in normal abduction is logically inderivable and empirically indecidable in rigorous logical sense. The major concern in this paper is not its distinction from induction and deduction but the difference of physical law from not so rigorous abduced objects. With a logically rigorous analysis, case studies in this paper will bring us a significantly different abductive reasoning manner regarding physical laws in Sections 2 and 3.

Tracing back to earlier times, Hume's problem also mixed natural laws with normal empirical inductions and denied any certainty of natural laws, of course, including physical laws. It was an incisive question for natural law for more than two and half centuries, Hume [15][16], and was left unsolved up to the present time, both in classical reasoning and in non-standard abductive reasoning. The fatal problem is how to know the conformability of past and future for a natural law in any empirical test, which requires infinite empirical induction. From Hume's time, philosophers and logicians had made great efforts to rescue the certainty in relation to the empirical origin of natural laws and finally laid out two important and roundabout but unsuccessful tactics in modern times to counter fight Hume's skepticism. One was to justify the feasibility or reliability of an empirical inductive procedure for yielding a natural law, and the other was to dissolve the skeptical threat by cancelling the core sense of Hume's problem. In the first tactic, some eventually drew support from the probability argument to avoid the problem of natural law's certainty, e.g., Fisher [17], Carnap [18-21], Reichenbach [22,23], and others showed the empirical inductive procedure was always more reliable than Hume's reasoning, e.g., Worrall [24], Kelly [25]. In the second tactic, Popper [26-29] used falsification to replace verification, Shier [30] used the unsolvability of Hume's problem in a three-valued logical schema to evade Hume's critique, and etc., in which they actually used empirical inductive falsification or empirical inductive unsovability to replace empirical inductive verification, and still concentrated on empirical induction to find a resolution of Hume's problem. The two tactics all sank into the circular dilemma of using induction to justify induction which Hume had already denounced far earlier, see Howson [31]. All these arguments are unsuccessful to thoroughly free the natural law system from Hume's critique, see Skyrms [32], Howson [31], and, especially, are unable to surpass the problem Hume outlined and to bring enterprising studies to the natural law system to a level higher than what Hume had achieved. Up to the present time, as a methodological problem in natural science, the core issue unsolved in Hume's radical skepticism is always the problem of infinite induction, e.g., see Boulter [33], Okasha [34], Hetherington [35], Schurz [36]. Different from the above two tactics, this paper will open up a new route to deal with Hume's problem in a recursive pattern to overcome the problem of infinite induction.

Another development from the discussions of Hume's problem is Goodman's discovery [37,38]. Goodman proposed a triple propositional category of accidental, lawlikeness, and natural law to deal with the analysis involving Hume's problem, in which only lawlike propositions, but not all empirical propositions, are possible to 
serve as candidates for becoming a natural law. For a long time lawlikeness had been discussed, e.g., Carnap [19], Barker and Achinstein [39], Small [40], Ullion [41], Quine [42], Hesse [43], Skyrms [33], Israsel [44], Chart [45], Godfrey-Smith [46], Schwartz [47]. Nevertheless, how to characterize lawlikeness had made little progress and, in fact, fell into difficulties until recently. All efforts were seemingly destined to come back to the endless old argument for inductive procedures similar to those in Hume's problem. The present paper will not involve Goodman's and his successors' explanations of lawlikeness. But the idea of lawlikeness indicating a candidate for natural law is adopted to make up an important logical judgment leading to natural law.

The paper is organized as follows: Section 2, first clarifies the realistic logical path for yielding a physical law by case studies in the mathematical logic framework, discusses the relation among characteristics of normal abduction, lawlikeness, and physical law, gets rid of some long-term misunderstandings, and finally introduces the new abductive framework, called law-deriving abduction. Section 3 defines recursive abduction in logical and operational senses and discusses the universality of physical law. Section 4 gives a conclusive solution to Hume's problem based on the framework of recursive abduction. Section 5 summarizes the main conclusions.

\section{Analysis on Physical Laws Derived from Experiments}

In order to precisely re-clarify the objective ground on which the physical law is founded, we first focus on the case study of some physical laws in mathematical logical form.

\subsection{Case 1: Galilean Derivation for the Inertia Motion}

Fig. 1 Illustration of Galilean inference in his slant experiment

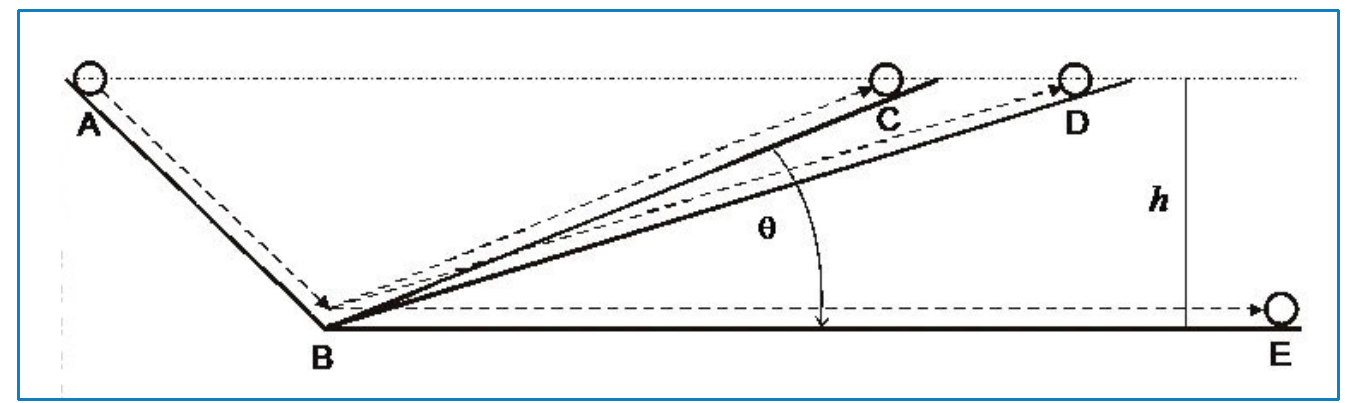

Galilean discovery, Galilei [48], can be illustrated in Fig. 1. First, a metal ball freely moves down from "A" to "B" along slant "A-B", proceeds to move up to "C" along slant "B-C", and during moving on "B-C", it slows down to zero at the highest point " $\mathrm{C}$ "; if one neglects the resistance, the heights of points " $\mathrm{A}$ " and " $\mathrm{C}$ " are equivalent $h$. Second, keeping the point "B" fixed and turning slant "B-C" to "B-D", and repeating the process in the first time, the metal ball reaches the final point "D" along fold line "A-B-D"; if one neglects the resistance, the height of point " $\mathrm{D}$ " is also $h$. That is, turning the slant between $\angle \mathrm{CBE}$ has no impact on the height $h$. So far, we induce from the slant experiment that the metal ball will arrive at $h$ and slows down to zero at its terminal point on the slant B-C for any $\theta$ between $\angle \mathrm{CBE}$. Galilei further thought that if the slant "B-C" turns to close the horizontal plane more and more, to reach the height $h$ the variation of the velocity of the metal ball will become smaller and smaller gradually, and finally if one lets the slant "B-C" infinitely close to the horizontal plane "B-E", to reach the final height $h$ the metal ball will always have to move on and therefore to keep its velocity constant. So, Galilei concluded that if there is not any external force imposed on an object, the object will keep its velocity constant to move horizontally. It is the preliminary description of inertia motion which led to Newton's inertia law 
eventually in a more general form.

Restate Galilean discovery more rigorously in the form of propositional logic as follows:

1) Neglect the resistance, described as $e$.

2) For all observable $\theta$ between $\angle \mathrm{CBE}$, if a metal ball freely moves down from the height $h$ and along the slant "A-B" from "A" to "B", described as $h_{\downarrow}$, it will get the maximal velocity $v_{0}$ at "B", described as $v_{0}$, and proceeds to move up along the slant "B-C" and finally reaches the height $h$ too, described as $h_{\uparrow}$, during which $v_{0}$ slows down to zero, described as $v_{0 \downarrow}:\left(h_{\downarrow} \rightarrow v_{0}\right) \rightarrow\left(e \rightarrow h_{\uparrow} \wedge v_{0 \downarrow}\right)$. The inductive conclusion $p_{I}$ from the experiment is $p_{I}=e \rightarrow h_{\uparrow} \wedge v_{0 \downarrow}$.

3) As a reasonable supposition, change angle $\theta$ such that the slant "B-C" infinitely tends to coincide with horizontal "B-E", described as $\theta_{B E}, h_{\downarrow}$ will also result $h_{\uparrow}$, and to do so unless the metal ball keeps $v_{0}$ constant moving on infinitely along "B-E", described as $\neg v_{0} \rightarrow \neg h_{\uparrow}$, thus, the metal ball will keep $v_{0}$ constantly moving on along "B-E", described as $h_{\uparrow} \rightarrow\left(e \rightarrow v_{0}\right): \theta_{B E} \rightarrow\left(\left(\neg\left(e \rightarrow v_{0}\right) \rightarrow \neg h_{\uparrow}\right) \rightarrow\left(h_{\uparrow} \rightarrow\left(e \rightarrow v_{0}\right)\right)\right)$.

4) Summing up from “1)" “3)", we obtain

$$
\left(\left(h_{\downarrow} \rightarrow v_{0}\right) \rightarrow\left(e \rightarrow h_{\uparrow} \wedge v_{0 \downarrow}\right)\right) \rightarrow\left(\theta_{B E} \rightarrow\left(\left(\neg\left(e \rightarrow v_{0}\right) \rightarrow \neg h_{\uparrow}\right) \rightarrow\left(h_{\uparrow} \rightarrow\left(e \rightarrow v_{0}\right)\right)\right) .\right.
$$

Concluding from (2.1),

$$
\theta_{B E} \rightarrow\left(\left(\neg\left(e \rightarrow v_{0}\right) \rightarrow \neg h_{\uparrow}\right) \rightarrow\left(h_{\uparrow} \rightarrow\left(e \rightarrow v_{0}\right)\right)\right) .
$$

(2.1) completely describes Galilean analysis and (2.2) is the right-hand side in (2.1). (2.2) is a tautology, described as $\mathcal{L}_{G}$, called the Galilean "quasi-law tautology" (in the case without specific assignment, described as $\mathcal{L}$ ). If we are merely concerned with the conclusion, (2.1) and (2.2) are equivalent. However, (2.2) cannot be directly tested in the slant experiment, and it is the result of tautological inference from the experimentally observable process under Galilean premise $h_{\uparrow}$. Only the left-hand side in (2.1), described as $I$, is an inductive examination, and can be tested by the experiment. The inductive conclusion in $I$ is $p_{I}=e \rightarrow\left(h_{\uparrow} \wedge v_{0 \downarrow}\right)$. (2.1) includes all contents covering the experimentally observable processes and the tautological inference in Galilean analysis. It is neither a pure empirical induction nor a pure tautological inference but some kind of combination of them to enable Galilei to derive his conclusion.

(2.1) is a tautology too, described as $\mathcal{T}_{G}$ and called a Galilean "lawlike tautology" (in the case without specific assignment, described as $\mathcal{T}$ ). The last formula $e \rightarrow v_{0}$ in $\mathcal{L}_{G}$, called inertia motion, is the Galilean conclusion. However, we cannot accept it as a physical law only based on the analysis of the slant experiment, for it is not the unique reasonable conclusion from the empirical inductive conclusion $p_{I}$. Galilei only used $h_{\uparrow}$ from the inductive conclusion $e \rightarrow h_{\uparrow} \wedge v_{0 \downarrow}$, and did not use $v_{0 \downarrow}$ from it. Galilean analysis does not include all conclusions objectively implied in the slant experiment.

We might convert our theoretical stand to agree with Aristotle's claim. From Aristotle, the basic empirical evidence contained in this experimental induction should be that the metal ball always naturally stops in the midway and never always freely moves on, Aristotle [49], that is, in the inductive conclusion $e \rightarrow h_{\uparrow} \wedge v_{0 \downarrow}, v_{0 \downarrow}$ but not $h_{\uparrow}$ is Aristotle's premise for the further analysis. Hence, from Aristotle's ideas $h_{\uparrow}$ is impossible to happen in $\theta_{B E}$ for it needs $\neg v_{0 \downarrow}$. Following Aristotle, (2.1) should become

$$
\left(\left(h_{\downarrow} \rightarrow v_{0}\right) \rightarrow\left(e \rightarrow h_{\uparrow} \wedge v_{0 \downarrow}\right)\right) \rightarrow\left(\theta_{B E} \rightarrow\left(\left(h_{\uparrow} \rightarrow \neg v_{0 \downarrow}\right) \rightarrow\left(v_{0 \downarrow} \rightarrow \neg h_{\uparrow}\right)\right)\right),
$$

and correspondingly (2.2) becomes 


$$
\theta_{B E} \rightarrow\left(\left(h_{\uparrow} \rightarrow \neg v_{0 \downarrow}\right) \rightarrow\left(v_{0 \downarrow} \rightarrow \neg h_{\uparrow}\right)\right.
$$

Similar to (2.1) and (2.2), (2.3) and (2.4) are also tautologies. But (2.3) and (2.4) conclude that when the slant "B-C" infinitely tends to coincide with the horizontal plane "B-E", the metal ball will stop in the midway and it will be impossible to reach the height $h$. The conclusion from (2.3) or (2.4) is opposite to that from (2.1) or (2.2). Describing $\mathcal{L}_{A}$ as the right-hand side in (2.3), a quasi-law tautology, and commonly denoting $I$ as the left-hand sides in (2.1) and (2.3) (notice: they are the same), similar to (2.1), (2.3) is a lawlike tautology, described as $\mathcal{T}_{A}$, and $I$ in $\mathcal{T}_{A}$ is the initial empirical induction. The last formula $\neg h_{\uparrow}$ in $\mathcal{L}_{A}$ is Aristotle's conclusion.

It is evident, the empirical induction in the slant experiment cannot determine which of $e \rightarrow v_{0}$ or $\neg h_{\uparrow}$ to be a physical law. They are merely the candidates for a physical law so far. Galilean $e \rightarrow v_{0}$ and Aristotle's $\neg h_{\uparrow}$ are two typical lawlike propositions, denoted as $p_{L}$. In $\mathcal{L}_{G}, h_{\uparrow}$ combining with the particular logical consistent explanation turns out $e \rightarrow v_{0}$ and excludes $v_{0 \downarrow}$, while, in $\mathcal{L}_{A}, v_{0 \downarrow}$ combining with the other particular logical consistent explanation turns out $\neg h_{\uparrow}$ and excludes $h_{\uparrow}$, although the experimental inductive conclusion $p_{I}=e \rightarrow h_{\uparrow} \wedge v_{0 \downarrow}$ is the same for both. The difference in logical consistent explanations, respectively indicated by $\mathcal{L}_{G}$ and $\mathcal{L}_{A}$, plays an important role to determine different candidates of a physical law. The consistent explanation presented in a quasi-law tautology is an indispensable logical ground to construct a lawlike proposition, and the initial inductive observation in the slant experiment only offers the reasoning clue for inspiring scientists to suggest their own lawlike propositions.

The initial experimental induction cannot uniquely exclude one and support another in choosing between $\mathcal{L}_{G}$ and $\mathcal{L}_{A}$. Only a further empirical test is possible to determine which of them to be a physical law. In history, the further corollaries from Galilean inertia motion, such as parabolic motion, Galilean transformation in the inertial system, static phenomena in the rotating earth, and etc., contributed further empirical supports to make the Galilean lawlike proposition eventually become a natural law. Whereas Aristotle's law of motion was rejected for its corollaries, except the static phenomena of the earth's rotation, were not supported by further empirical observations. They involve nothing of Kuhn's incomparable paradigms, Kuhn [50]. Abduction offers the litmus test for their qualities.

Taking parabolic motion as an example, and denoting $g$ as the acceleration of gravity and $m_{p}$ parabolic motion, we have

$$
\left(e \rightarrow v_{0}\right) \rightarrow\left(\left(e \rightarrow v_{0}\right) \wedge g \rightarrow m_{p}\right)
$$

in which $\left(e \rightarrow v_{0}\right) \wedge g \rightarrow m_{p}$ gives a new empirical inductive observation, described as $p_{I}^{\prime}$. The judgment to (2.5) supporting Galilean inertia motion has the reasoning schema of

$$
p_{I}^{\prime},\left(p_{L} \rightarrow p_{I}^{\prime}\right) / p_{L}
$$

a typical abduction, where, $p_{I}^{\prime}=\left(\left(e \rightarrow v_{0}\right) \wedge g \rightarrow m_{p}\right)$ and $p_{L}=\left(e \rightarrow v_{0}\right)$.

As is well-known in abduction, all natural laws have this feature: in Einstein's relativity, it is principle of the constancy of the velocity of light is used to interpret the Michelson-Morley experiment but not vice versa, meanwhile the Michelson-Morley experiment gives empirical truth to the principle of constancy of light velocity; in Darwin's evolution theory, it is principle of natural selection is used to interpret the phenomena of the evolution of species but not vice versa, meanwhile the phenomena of species' evolution gives empirical truth to the principle of natural selection; and so on. They finally become natural laws all through $p_{I}^{\prime},\left(p_{L} \rightarrow p_{I}^{\prime}\right) / p_{L}$, see, e.g., 
Pombo and Gerner [7]. Here the further issue is why physical laws are always most excellent in their universality compared with other empirical sciences and commonsense.

\subsection{Further Discussions on the Abduction for Deriving Physical Laws}

Describing $\mathcal{L} p_{L}$ as a tautology $\mathcal{L}$ containing conclusion $p_{L}$, we define quasi-law tautologies, lawlike propositions, and natural laws as follows:

Definition 1. $\mathcal{L} p_{L}$ is a quasi-law tautology if the corollary of $p_{L}$ is an empirical induction $p_{I}^{\prime}$, and $p_{L}$ is called a lawlike proposition.

Definition 2. $p_{L}$ is a natural law, described as $p_{L}^{t}$, if and only if $p_{I}^{\prime}$, the corollary of $p_{L}$, is empirically true.

In Definition 2, $p_{L}$ and $p_{L}^{t}$ are the same in terms of their sentence contents, but they are distinguished by the different empirical truth value statuses. $p_{L}$ is merely the conclusion in $\mathcal{L} p_{L}$ without an empirical truth value but $p_{L}^{t}$ is by abduction the empirically valued $p_{L}$.

In Case 1 , Galilean $p_{L}=e \rightarrow v_{0}$ in $\mathcal{L}_{G}$ obtains empirical truth by abduction in $p_{L} \rightarrow p_{I}^{\prime}$ to become a natural law $p_{L}^{t}$, whereas Aristotle's $p_{L}=\neg h_{\uparrow}$ in $\mathcal{L}_{A}$ fails to obtain empirical truth by abduction in $p_{L} \rightarrow p_{I}^{\prime}$ and is rejected as a natural law.

Using the symbols in Definitions 1 and 2, we always denote abduction for deriving a physical law as follows

$$
p_{I}^{\prime},\left(p_{L} \rightarrow p_{I}^{\prime}\right) /\left(p_{L}=p_{L}^{t}\right)
$$

It is still an abduction of course, in which we discriminate $p_{L}$ and $p_{L}^{t}$, a lawlike proposition and a physical law. A lawlike proposition is the conclusion in quasi-law tautology $\mathcal{L} p_{L}$, and a natural law is a lawlike proposition obtaining the empirical truth in abduction. The form of (2.6) slightly differs from the current abductive reasoning schema and emphasizes the different empirical truth statuses of $p_{L}$ and $p_{L}^{t}$.

\subsection{A New Kind of Abduction}

(2.6) indicates a new kind of abduction, called law-deriving abduction, briefly L-abduction. It means that normal abduction in $p \rightarrow p_{I}^{\prime}$ is merely necessary but not sufficient for deriving a physical law. Normal abduction cannot sufficiently derive any strict natural law such as physical law. On the other hand, what is revealed is that the combination of quasi-law tautology, indicating the lawlikeness following Definition 1, with empirical abduction, indicating the empirical test following Definition 2, gives the sufficient derivability of a physical law, though empirical abduction alone is always insufficient to do it. This new kind of abduction introduces the new sufficiency for deriving a strict natural law, and is grounded on the combination of lawlikeness with empirical abduction. In order to establish sufficient abduction in empirical induction for deriving a physical law we should focus on the combination of lawlikeness with empirical abduction but not normal abduction alone.

In terms of the simplest case in which there is only $p_{I}^{\prime}$, i.e., there is only one corollary of $p_{L}$ considered in abduction, the above analysis on Case 1 is generally summarized in (2.7) and (2.8),

$$
\left\{\begin{array}{l}
\left(p_{I} \rightarrow \mathcal{L} p_{L}\right) \rightarrow\left(p_{L} \rightarrow p_{I}^{\prime}\right) ; \\
p_{I}^{\prime},\left(p_{L} \rightarrow p_{I}^{\prime}\right) /\left(p_{L}=p_{L}^{t}\right) .
\end{array}\right.
$$


By similar analysis conducted in Case 1, it is easily to show that (2.7) and (2.8) describes the general reasoning process followed by strict natural laws such as Newtonian three laws of motion, Darwin's law of evolution, mass conservation law in chemistry, Mendeleev periodic law of elements, Mendel's law of inheritance, principle of constancy of light velocity, and so on. Every strict natural law first has its own lawlikeness indicated by a quasi-law tautology, in which particular consistent explanations are presented, and then, are supported by empirical observations in abduction.

The case study clearly reveals that a strict natural law is sufficiently but not fuzzily logically derivable in (2.7) and empirically decidable in (2.8). This result differs from the general view that the current concept of abduction gives us in the normal empirical inductive process. In the derivation for a strict natural law, abduction can bring us the greater certainty than is implied by the word of $f u z z y$.

So far, we have clarified at least that a lawlike proposition and a strict natural law are never a normal abductive result. In (2.7), only $p_{I}$ is a purely descriptive empirical result and it merely offers preliminary raw materials for inspiring scientists to find the lawlikeness for the candidate of natural law. It contributes the inspiration psychologically but neither the necessity nor the sufficiency for deriving a natural law. Moreover, (2.2) derived from (2.1) and (2.4) from (2.3) all obey $\left(p_{I} \rightarrow C\right) \vdash p_{L}$, where $C$ indicates an interpreting choice from $p_{I}$. We derive different lawlike results $p_{L}=e \rightarrow v_{0}$ or $p_{L}=\neg h_{\uparrow}$ by introducing the interpretation $h_{\uparrow}$ or $v_{0 \downarrow}$ as $C$. Initial empirical observation in $I$ at most delivers $p_{I}$. Logically irrelevant to $p_{I}$, the logical consistent explanation in $\mathcal{L}$, rather than the great number of cumulative evidences in the normal empirical abduction, yields a lawlike proposition. Lawlike tautology $\mathcal{T}$ asks both a consistent logical relationship and a set of observables. Based on $\mathcal{L}$ in $\mathcal{T}$ but not $I$ in $\mathcal{T}$, the candidate of a natural law is eventually proposed.

Theoretically, the initial empirical induction $I$ is unnecessary, and it is only a psychological factor to inspiring scientists. For example, more than two-thousand and three-hundred years ago Aristotle proposed his motion law without the empirical inductive result $p_{I}=e \rightarrow\left(h_{\uparrow} \wedge v_{0 \downarrow}\right)$ in a slant experiment. $\mathcal{L} p_{L}$ and $p_{I}^{\prime}$ together consist of sufficiency to abduct $p_{L}^{t}$. So (2.7) can be simplified into (2.9) as the following

$$
\left\{\begin{array}{l}
\mathcal{L} p_{L} \rightarrow\left(p_{L} \rightarrow p_{I}^{\prime}\right) ; \\
p_{I}^{\prime},\left(p_{L} \rightarrow p_{I}^{\prime}\right) /\left(p_{L}=p_{L}^{t}\right) .
\end{array}\right.
$$

Henceforth, we always refer $L$-abduction to (2.9) and (2.10).

The case study clearly reveals that a strict natural law is logically derivable in (2.9). It is an important feature differing from normal abduction. (2.9) formally distinguishes a strict natural law from a commonsense. The next issue left is how the empirical truth of a strict natural law is decidable in observational test from the further analysis on (2.10).

It is concluded now that

1. Beginning with an initial empirical inductive conclusion $p_{I}$, one cannot determine $p_{L}$ unless it is associated with a consistent relationship presented in quasi-law tautology $\mathcal{L}$; and $p_{L}$ is the conclusion in $\mathcal{L}$, namely $\mathcal{L} p_{L}$.

2. $p_{L}$ cannot become $p_{L}^{t}$ unless its corollaries $p_{I}^{\prime}, p_{I}^{\prime \prime}, \ldots$ are empirically true.

3. Not the initially inspiring $p_{I}$ but the combination of lawlikeness with following empirical inductive $p_{I}^{\prime}$, $p_{I}^{\prime \prime}, \ldots$ gives the sufficiency to infer a natural law. It is an L-abduction.

4. (2.9) and (2.10) completely define the L-abduction.

Normal abduction always prepares well for non-monotonicity and dynamic reasoning but overlooks the sufficiency of derivation for strict natural law. Normal abduction as a general concept is certainly over-pessimistic. 
It is necessary to distinguish $L$-abduction from normal abduction. Their difference can be summarized as: the normal abduction deals with loose phenomena of $A$ and $B$ in a speculative $A \rightarrow B$, just like a physician facing a patient to conjecture what is the explanation of the patient's symptom, in which usually no sufficiency exists for deriving a conclusion, Meheus and Batens [2]; $L$-abduction deals with the logically compact relation between $p_{L}$ and $p_{I}^{\prime}$ consistently based on $\mathcal{L} p_{L}$ and the empirical truth of $p_{I}^{\prime}$, just like one using originally consistent geometry in the measurement of realistic space to determine whether the conclusions from the geometrical theorems are empirically feasible, in which there is the sufficient information to derive the conclusion.

\subsection{Case 2: Difference between Empirical Inductive Conclusion and Physical Law}

Is the sentence "the sun rises from the east everyday" an empirical inductive conclusion (purely as a repeated result from numerous empirical observations) or a physical law (as an empirically true corollary from inertia rotation of the earth following inertia law)? The difference between a physical law and a normal empirical inductive result is not their empirical contents but their logical consistent explanations presented in a quasi-law tautology. Empirical observation at most produces inductive conclusions, only $L$-abduction possibly produces a strict natural law. Here, three things should be distinguished: empirical induction, normal abduction, and $L$-abduction. If "Tom always dresses in red colors" is concluded from the observable result of "Tom dressed in red colors every day up to now", this is an empirical inductive conclusion. If from "Tom likes to dress in red colors" one infers "Tom always dresses in red colors", this is normal abductive reasoning, for like and dress are different predicates. However, it will be impossible to derive a lawlike proposition and, further, a strict natural law, unless a quasi-law tautology can be applied to making sense explaining Tom's dressing in a consistent manner. Only $L$-abduction is possible to finally yield a strict natural law.

\subsection{Case 3: Galilean Law of Free Fall}

Aristotle regarded from the intuitive impression that the heavier a body $\left(w^{\uparrow}\right)$ is, the faster its free fall $\left(g^{\uparrow}\right)$ is, and, naturally, the lighter a body $\left(w^{\downarrow}\right)$ is, the slower its free fall $\left(g^{\downarrow}\right)$ is: $\left(w^{\uparrow}>w^{\downarrow}\right) \rightarrow\left(g^{\uparrow}>g^{\downarrow}\right)$. By using reduction to absurdity, Galilei refuted Aristotle's viewpoint, Galilei, [48]: Supposing Aristotle's assertion right, if a heavier body is bundled with a lighter one, the total weight $w^{\uparrow \downarrow}$ will increase to their added value $w^{\uparrow \downarrow}=\left(w^{\uparrow}+\right.$ $\left.w^{\downarrow}\right)>w^{\uparrow}$, and if they together freely fall, then their free fall $\left(g^{\uparrow \downarrow}\right)$ will be faster than $g^{\uparrow}$ :

$$
\left(w^{\uparrow}+w^{\downarrow}\right) \rightarrow\left(g^{\uparrow \downarrow}>g^{\uparrow}\right)
$$

on the other hand, if a faster falling heavier body is bundled with a slower falling lighter one, then the slower one will slow down the faster one to result in their common free fall $\left(g^{\uparrow \downarrow}\right)$ slower than $g^{\uparrow}: g^{\uparrow \downarrow}<g^{\uparrow}$, that is,

$$
\left(w^{\uparrow}+w^{\downarrow}\right) \rightarrow\left(g^{\uparrow \downarrow}<g^{\uparrow}\right)
$$

combining (2.11) with (2.12), obtain

$$
\left(w^{\uparrow}+w^{\downarrow}\right) \wedge\left(g^{\uparrow} \wedge g^{\downarrow}\right) \rightarrow\left(g^{\uparrow \downarrow}>g^{\uparrow}\right) \wedge\left(g^{\uparrow \downarrow}<g^{\uparrow}\right) ;
$$

and it is contradictory. And the contradiction is avoided unless $g^{\uparrow}=g^{\downarrow}$, that is, the free falls of heavier and lighter bodies are the same. Galilei therefore proposed $g^{\uparrow}=g^{\downarrow}$ by the following reasoning 


$$
\left(\neg\left(g^{\uparrow}=g^{\downarrow}\right) \rightarrow\left(\left(g^{\uparrow \downarrow}>g^{\uparrow}\right) \wedge\left(g^{\uparrow \downarrow}<g^{\uparrow}\right)\right)\right) \rightarrow\left(\neg\left(\left(g^{\uparrow \downarrow}>g^{\uparrow}\right) \wedge\left(g^{\uparrow \downarrow}<g^{\uparrow}\right)\right) \rightarrow g^{\uparrow}=g^{\downarrow}\right) .
$$

(2.14) is a quasi-law tautology, in which $g^{\uparrow}=g^{\downarrow}$ is the conclusion, a lawlike proposition $p_{L}$. In this case, the empirical inductive result is $p_{I}=\left(g^{\uparrow}>g^{\downarrow}\right) \rightarrow\left(\left(w^{\uparrow \downarrow}>w^{\uparrow}\right) \rightarrow\left(g^{\uparrow \downarrow}>g^{\uparrow}\right)\right) \wedge\left(\left(g^{\uparrow} \wedge g^{\downarrow}\right) \rightarrow\left(g^{\uparrow \downarrow}<g^{\uparrow}\right)\right)$, which is omitted in (2.14) to simplify the expression. Galilean reasoning in (2.14) led to the discovery of the gravity acceleration constant $g$ on the earth's surface. Here $g$ is $p_{I}^{\prime}$ derived from a Galilean lawlike proposition and supports the law of free fall in abduction, namely $p_{I}^{\prime},\left(p_{L} \rightarrow p_{I}^{\prime}\right) / p_{L}=p_{L}^{t}$. It is an example without interpreting choice by dividing $p_{I}$. The formulae (2.9) and (2.10) still follow. If Galilei proposed $g^{\uparrow}=g^{\downarrow}$ merely from the said experimental observation of the free falling bodies in Leaning Tower of Pisa without the consistent explanation in (2.14), it would had been called an equal free falling phenomenon, a pure empirical inductive conclusion, but not the law of free fall. The consistent explanation in $\mathcal{L}$ is the indispensible character of a lawlike proposition preparing for a natural law.

2.6. Relationship between the Empirical Truth Values of $\mathcal{L} p_{L}$ and $p_{L}$

A tautology is always true for its semantics of connectives, namely it is connective-semantically true, but its contents may be empirically true or false. In Case 1, the contents of $\mathcal{L}_{G}$ are empirically true revealing the realistic empirical process, whereas $\mathcal{L}_{A}$ empirically false involving nothing of the realistic empirical process. It enlightens us that a quasi-law tautology $\mathcal{L} p_{L}$ is empirically true or false because $p_{L}$ is empirically true or false, and vice versa. Below, we proof this result as Theorem 1 in the general sense.

Theorem 1. $\left(p_{L} \rightarrow p_{I}^{\prime}\right) \leftrightarrow\left(\mathcal{L} p_{L} \rightarrow p_{I}^{\prime}\right)$

Proof of Theorem 1:

There are three steps in the proof:

Step 1, proof for $\left(\mathcal{L} p_{L} \rightarrow\left(p_{L} \rightarrow p_{I}^{\prime}\right)\right) \rightarrow\left(\mathcal{L} p_{L} \rightarrow p_{I}^{\prime}\right)$ :

(1) $\left(\mathcal{L} p_{L} \rightarrow\left(p_{L} \rightarrow p_{I}^{\prime}\right)\right) \rightarrow\left(\left(\mathcal{L} p_{L} \rightarrow p_{L}\right) \rightarrow\left(\mathcal{L} p_{L} \rightarrow p_{I}^{\prime}\right)\right)$

Axiom

(2) $\mathcal{L} p_{L} \rightarrow\left(p_{L} \rightarrow p_{I}^{\prime}\right)$

(3) $\left(\mathcal{L} p_{L} \rightarrow p_{L}\right) \rightarrow\left(\mathcal{L} p_{L} \rightarrow p_{I}^{\prime}\right)$

(4) $\mathcal{L} p_{L} \rightarrow p_{L}$ $\mathcal{L} p_{L}:=\mathcal{L}$ containing conclusion $p_{L}$

(5) $\mathcal{L} p_{L} \rightarrow p_{I}^{\prime}$ (3) (4) MP

Step 2, proof for $\left(\mathcal{L} p_{L} \rightarrow\left(p_{L} \rightarrow p_{I}^{\prime}\right)\right) \rightarrow\left(p_{L} \rightarrow p_{I}^{\prime}\right)$ :

(1) $\left(\mathcal{L} p_{L} \rightarrow\left(p_{L} \rightarrow p_{I}^{\prime}\right)\right) \rightarrow\left(p_{L} \rightarrow p_{I}^{\prime}\right)$

(2) $\mathcal{L} p_{L} \rightarrow\left(p_{L} \rightarrow p_{I}^{\prime}\right)$

(3) $p_{L} \rightarrow p_{I}^{\prime}$

Step 3, proof for $\left(\mathcal{L} p_{L} \rightarrow p_{I}^{\prime}\right) \leftrightarrow\left(p_{L} \rightarrow p_{I}^{\prime}\right)$ :

(1)" $\left(\left(\mathcal{L} p_{L} \rightarrow\left(p_{L} \rightarrow p_{I}^{\prime}\right)\right) \rightarrow\left(\mathcal{L} p_{L} \rightarrow p_{I}^{\prime}\right)\right) \wedge\left(\left(\mathcal{L} p_{L} \rightarrow\left(p_{L} \rightarrow p_{I}^{\prime}\right)\right) \rightarrow\left(p_{L} \rightarrow p_{I}^{\prime}\right)\right) \rightarrow\left(\mathcal{L} p_{L} \rightarrow p_{I}^{\prime}\right) \leftrightarrow\left(p_{L} \rightarrow p_{I}^{\prime}\right)$

Theorem

(2)" $\left(\left(\mathcal{L} p_{L} \rightarrow\left(p_{L} \rightarrow p_{I}^{\prime}\right)\right) \rightarrow\left(\mathcal{L} p_{L} \rightarrow p_{I}^{\prime}\right)\right) \wedge\left(\left(\mathcal{L} p_{L} \rightarrow\left(p_{L} \rightarrow p_{I}^{\prime}\right)\right) \rightarrow\left(p_{L} \rightarrow p_{I}^{\prime}\right)\right)$

Step 1 and Step 2

(3)" $\left(\mathcal{L} p_{L} \rightarrow p_{I}^{\prime}\right) \leftrightarrow\left(p_{L} \rightarrow p_{I}^{\prime}\right)$

(1)'(2)'MP

Denoting empirically true $\mathcal{L} p_{L}$ as $\mathcal{L} p_{L}{ }^{t}$, we have 
Corollary 1. $\mathcal{L} p_{L}^{t} \equiv p_{L}^{t}$.

In $\mathcal{L} p_{L}{ }^{t}$ and $p_{L}^{t}$ in Corollary 1 , superscript $t$ indicates the empirical truth of $\mathcal{L} p_{L}$ and $p_{L}$ obtained from $L$-abduction. The meanings of Corollary 1 are only that the empirical truth value of $\mathcal{L} p_{L}$ is equivalent to the empirical truth value of $p_{L}$ obtained from $L$-abduction, but not $\mathcal{L} p_{L} \equiv p_{L}$.

Proof of Corollary 1: From Theorem 1, if $p_{L}$ obtains empirical truth by $L$-abductive reasoning in $p_{L} \rightarrow p_{I}^{\prime}$ to become $p_{L}^{t}$, then $\mathcal{L} p_{L}$ will obtain empirical truth by $L$-abductive reasoning in $\mathcal{L} p_{L} \rightarrow p_{I}^{\prime}$ to become $\mathcal{L} p_{L}{ }^{t}$, and vice versa.

Corollary 1 reveals a physical law $p_{L}^{t}$ must correspond to an empirically true $\mathcal{L} p_{L}$. It explicitly appeals to us, besides the connective-semantically truth of a tautology, to concentrate on the empirical truth value of the sentence's contents in quasi-law tautology $\mathcal{L} p_{L}$ though it is never the subject in classical formal logic. Empirically true $\mathcal{L} p_{L}$ is always meaningful in physics. In Case $1, \mathcal{L}_{G}$ implies the conservation of mechanical energy, in which the summation of potential energy, indicated by $h_{\uparrow}$ of the metal ball, and kinetic energy, indicated by $v_{0}$ of the metal ball, keeps constant. It was clearly proposed almost one-hundred and fifty years later from Galileo's time. Also, in Case 3, the empirically true quasi-law tautology (2.14) implies the equivalence of gravitational mass and inertial mass, which was unambiguously proposed almost three-hundred years later. They have revealed the importance of the empirical truth value of a quasi-law tautology. Classical formal logic involves nothing such as whether or not the contents of a tautology are empirically true and needs not discriminate the connective-semantic truth and empirical truth. However, derivation of a physical law needs such a discrimination, and we must answer whether or not the contents of a quasi-law tautology are empirically true, just as we had seen in $\mathcal{L}_{G}$ and $\mathcal{L}_{A}$. Their empirical truth values are not inconsequential and always make sense in physics. This is another non-ignorable vital characteristic of $L$-abduction distinguishing it from classical logic. Usually we always pay attention to a natural law rather than to a quasi-law tautology. The analysis to quasi-law tautology may be neglected too much in physical studies.

\section{Recursive Abduction Based on Quasi-Law Tautology}

\subsection{A Comparison between Physics and Geometry}

Physics as empirical science and geometry as a formal system are always viewed as essentially different knowledge fields. However, under the viewpoint of $L$-abduction presented by (2.9) and (2.10), they are the same.

All theorems of geometry are just lawlike propositions and have the same logical status as the candidates of physical law $p_{L}$ without any particularity until they are tested in empirical observation by $L$-abduction. The logical procedure for yielding the theorems of geometry is also $\mathcal{L} p_{L}$, in which the relation between axiom and theorem is presented. When they pass the empirical test by $L$-abduction, they become natural law, and the complete logical procedure is the combination of (2.9) and (2.10) as well. This logical procedure is indifferent between physical law and geometrical theorem. Geometry as the feasible descriptive system to the realistic world differs from physics merely by only being given usually without reference to specific $I$ in $\mathcal{T}$, a non-essential feature for physical laws. The axioms of geometry or the fundamental explanations of physical laws all are presented in $\mathcal{L} p_{L}$; geometrical theorems and physical laws such as Newtonian motion laws, Einstein's principle of constancy of light velocity, and so on, all correspond to the conclusions in $\mathcal{L} p_{L}$, unlike the current 
misunderstanding about correspondence between physical laws and axioms in geometry. Obviously in $L$-abduction, the physical law corresponds to the theorem of geometry, and the relationship between geometrical theorem and corresponding axiom is presented in $\mathcal{L} p_{L}$.

In $\mathcal{T}$, the antecedent $I$ is an initial empirical induction inspiring the scientist to propose a lawlike proposition, it is merely to describe the most feasible discovery manner in practice for the scientist who always makes the effort to eliminate psychological illusions to stimulates directly insight into objective nature. Theoretically, the scientist can directly uses observables to construct a quasi-law tautology $\mathcal{L} p_{L}$ without the initial specific empirical induction $I$ in $\mathcal{T}$, and in this case, the lawlike proposition directly derived from $\mathcal{L} p_{L}$ will likewise become a natural law as long as its logical corollary $p_{I}^{\prime}$ is empirically true in $L$-abduction. Geometry as the description of realistic physical space is just such a natural law system. Without drawing support from specific induction $I$ in $\mathcal{T}$, geometers directly construct a set of consistent inferences (corresponding to a set of quasi-law tautologies) to set up a lawlike system, in which idealized spatial descriptive elements, such as point, line, plane, and so on, are organized in consistent logical contexts to compose possibly consistent spatial descriptions, such as Euclidean geometry and non-Euclidean geometries; if anyone of them infers true empirical results in $L$-abduction, it will become a natural law system. This process is completely similar to determining which of the conclusions in $\mathcal{L}_{G}$ and $\mathcal{L}_{A}$ into a natural law. In Newton's time, Euclidean geometry was a natural law system, and in Einstein's time, it became non-Euclidean geometry. Natural law systems are always verifiable and falsifiable in $L$-abduction.

Physics and geometry as descriptive systems applicable to the realistic world similarly contain two parts: a consistent formality part presented by the quasi-law tautology and an empirically tested part presented by the $L$-abduction. Traditionally, it was customary to concentrate more on the empirically tested part in physics and on the consistent formal part in geometry. $L$-abduction reveals to us that these two parts are equally important for physics and geometry. We shall now discuss what the quasi-law tautology in $L$-abduction has brought to physics.

\subsection{Recursive Abduction}

First, let us come back to (2.2). For time $T$, we can always define measurable recursive partitions $t_{k}, k=$ $0,1,2, \cdots$, such that

$$
T=\Sigma t_{k}, \quad k=0,1,2, \cdots
$$

The most familiar $t_{k}$ includes seconds, minutes, hours, days, months, years, centuries, and so on. When we choose time as a recursive dimension, the recursive feature of time is certainly treated as the ultimate attribute of time itself in the explanation given in a quasi-law tautology. What we want to clarify here is how recursion functions in $L$-abduction differently from in a normal abduction.

Describing $p_{L}$ in $t_{k}$ as $p_{L}^{t_{k}}, k=0,1,2, \cdots$, in $(2.2), p_{L}^{t_{k}}=\left(e \rightarrow v_{0}\right)^{t_{k}}$. From the tautological character of (2.2), naturally,

$$
\left(\left(\neg\left(e \rightarrow v_{0}\right) \rightarrow \neg h_{\uparrow}\right) \rightarrow\left(h_{\uparrow} \rightarrow\left(e \rightarrow v_{0}\right)\right)\right) \rightarrow\left(\left(\neg\left(e \rightarrow v_{0}\right) \rightarrow \neg h_{\uparrow}\right) \rightarrow\left(h_{\uparrow} \rightarrow\left(e \rightarrow v_{0}\right)^{t_{k}}\right)\right) .
$$

(3.1) means that as the tautological result the conclusion in (2.2) will always be original in any $t_{k}$. It only presents the request from the logical consistent explanation given in (2.2), and is purely a formality so far. It is not a positive result in empirical world, for example, Aristotle's empirically false $\left(\neg h_{\uparrow}\right)^{t_{k}}$ also holds in $\mathcal{L}_{A}$ similarly to (3.1).

However, following (3.1), the empirical test to $\left(e \rightarrow v_{0}\right)^{t_{k}}$ in (2.5) becomes (3.2) correspondingly 


$$
\left(e \rightarrow v_{0}\right)^{t_{k}} \rightarrow\left(\left(e \rightarrow v_{0}\right) \wedge g \rightarrow m_{p}\right)^{t_{k}}, \quad k=0,1,2, \cdots
$$

In (3.2), $\left(\left(e \rightarrow v_{0}\right) \wedge g \rightarrow m_{p}\right)^{t_{k}}$ as $p_{I}^{\prime}$ is an experimental observation happening in realistic $t_{k}$, and it is not a formality but a practice in the empirical world. (3.1) requires the empirical test to $e \rightarrow v_{0}$ in (2.2) conducted in the temporal series $t_{k}, k=0,1,2, \cdots$. In other words, an empirical test to $e \rightarrow v_{0}$ of (2.2) is requested recursively in real time. That is,

if

a) $\left(e \rightarrow v_{0}\right)^{t_{0}}$ is empirically true in (3.2) by $L$-abduction,

b) $\left(e \rightarrow v_{0}\right)^{t_{k}}$ is empirically true in (3.2) by $L$-abduction,

c) $\left(e \rightarrow v_{0}\right)^{t_{k+1}}$ is empirically true in (3.2) by $L$-abduction,

then

d) $\left(e \rightarrow v_{0}\right)$ in (2.5) will be empirically true in $t_{k}, k=0,1,2, \cdots$, namely, passes the empirical test in $L$-abduction;

and

e) otherwise, $\left(e \rightarrow v_{0}\right)$ in (2.5) will be false in $t_{k}, k=0,1,2, \cdots$, namely, fails in the empirical test in $L$-abduction.

For example, in Case 1, Galilean $e \rightarrow v_{0}$ obtains the empirical truth in $t_{k}, k=0,1,2, \cdots$, while Aristotle's $\neg h_{\uparrow}$ does not. This time-recursive requirement from a quasi-law tautology is generally the same for both physical laws and geometrical theorems used in realistic space description.

The above discussion is made in terms of time-recursive abduction. In fact, for any recursively measurable dimension we can introduce the recursive test in an $L$-abduction as long as $\mathcal{L} p_{L}$ is uncontradictory to this dimension. And in this case, we call such a dimension the suitable recursive dimension. Not all dimensions may be suitable recursive dimensions, e.g., all quantum dimensions are contradictory to $\mathcal{L}_{G}$ for $\mathcal{L}_{G}$ disagrees with Heisenberg's uncertainty relation.

Letting $r_{k}, k=0,1,2, \cdots$, be a suitable recursive dimension, we have the general definition of recursive abduction as follows:

Definition 3. A recursive abduction refers to:

if

a) $p_{L}^{r_{0}}$ is empirically true in $p_{L}^{r_{0}} \rightarrow p_{I}^{\prime r_{0}}$ by $L$-abduction,

b) $p_{L}^{r_{k}}$ is empirically true in $p_{L}^{r_{k}} \rightarrow p_{I}^{\prime r_{k}}$ by $L$-abduction,

c) $p_{L}^{r_{k+1}}$ is empirically true in $p_{L}^{r_{k+1}} \rightarrow p_{I}^{\prime r_{k+1}}$ by $L$-abduction,

then

d) $p_{L}$ in $p_{L} \rightarrow p_{I}^{\prime}$ will be empirically true in $r_{k}, k=0,1,2, \cdots$, and becomes $p_{L}^{t}$, namely, passes the empirical test in $L$-abduction;

and

e) otherwise, $p_{L}$ in $p_{L} \rightarrow p_{I}^{\prime}$ will be empirically false in $r_{k}, k=0,1,2, \cdots$, namely, fails in the empirical test in $L$-abduction.

Different from the mathematical recursive function, if Definition 3 is used in a physical measurement, the practical operational sense of $r_{k}$ and $r_{k+1}$ is that $k$ is randomly selected from $k=0,1,2, \cdots$. Namely, in physical 
measurement, we use randomness of $k$ in place of arbitrariness of $k$ in mathematics to make the recursion. The randomness interpretation of $k$ contains two meanings: first, it allows a new probability argument of natural law's universality; and second, it is just the recursive expression for the well familiar repeatability of experimental or empirical observations in physical studies.

Recursive abduction relies on the combination of (2.9) and (2.10), and impossibly comes true in classical logic and normal abduction. Recursive abduction reveals the essence of sufficiency for deriving a physical law in $L$-abduction. In other words, the universality of a physical law is nothing else but the physical law holding in one or more suitable recursive dimensions.

Recursive abduction can be used in all suitable recursive dimensions, such as space-recursion, mass-recursion, speed-recursion, and etc. For space-recursion, it refers to different locations in space; for mass-recursion, it refers to different quantities of mass; for speed-recursion, it refers to different speeds of motion; and so on. They not only consist of the verifiable universality of physical laws but also give the possibility to recursively falsify a physical theory in its inapplicable range, e.g., Newtonian mechanics was falsified in the space-recursion of micro field, in the mass-recursion of quantum field, and in the speed-recursion in high-speed field. Recursive abduction is the general character of physics system.

By the similar proof to Corollary 1, we have

Corollary 2. From Theorem 1 , if $p_{L}^{t}$ from $p_{L} \rightarrow p_{I}^{\prime}$ holds in $r_{k}, r=0,1,2, \cdots, \mathcal{L} p_{L}$ will be empirically true in $r_{k}, r=0,1,2, \cdots$.

Corollary 2 means that corresponding to $p_{L}^{t}$, a set of empirically true sentences in $\mathcal{L} p_{L}$ is consistent in $r_{k}, r=$ $0,1,2, \cdots$.

Such a set of sentences in $\mathcal{L} p_{L}{ }^{t}$ makes up the foundational explanation to $p_{L}^{t}$, and is meaningful in corresponding disciplines such as physics, geometry, or etc., as mentioned above. Those consistent sentences involve $p_{L}^{t}$ but usually contain more than $p_{L}^{t}$. They are usually distinct from $p_{L}^{t}$, and have their own analytical value.

From Definition 3, $L$-abduction is recursive as long as there is a suitable recursive dimension. The recursive $L$-abductive schema can be given as follows

$$
\left\{\begin{array}{l}
\mathcal{L} p_{L} \rightarrow\left(p_{L} \rightarrow p_{I}^{\prime}\right) ; \\
p_{I}^{\prime r_{0, k, k+1}}, \forall_{r_{k}}\left(p_{L}^{r_{k}} \rightarrow p_{I}^{\prime r_{k}}\right) /\left(p_{L}^{r_{0, k, k+1}}=p_{L}^{t r_{k}}\right), \quad k=0,1,2, \cdots
\end{array}\right.
$$

where $p_{I}^{\prime r_{0, k, k+1}}=\left\{p_{I}^{\prime r_{0}}, p_{I}^{\prime r_{k}}, p_{I}^{\prime r_{k+1}}\right\}$, and $p_{L}^{r_{0, k, k+1}}=\left\{p_{I}^{r_{0}}, p_{I}^{r_{k}}, p_{I}^{r_{k+1}}\right\}$.

(3.3) and (3.4) present an effective logical foundation for physical laws: the physical law is derivable in (3.3), and its empirical truth value is decidable in (3.4). Specifically, the universality of a natural law is formally derivable in quasi-law tautological sense and the empirical truth of a natural law is decidable in recursive abduction sense. Here the recursion is used as a feasible thinking manner grounded on natural number system.

In current probability arguments, the insuperable obstacle in Hume's problem is that the universality of natural law is produced from and, meanwhile, tested by a same empirical induction; if the induction confirms the universality, it must be infinitely carried on, thus, will be impossibly finished; all empirical inductions are doomed to be limited; in this way, any empirical test to the universal natural law only offers a limited inductive sample which always corresponds to a zero probability in the required infinite induction.

In the schema presented by (3.3) and (3.4), the things all have been changed. The universality of a strict natural law only refers to a requirement of logical consistence from a quasi-law tautology rather than it necessarily 
contains something equivalent to the unlimited universal applicability in the empirical world so that it needs the infinite empirical induction to support. The logical foundation for this universality is formed before, by no means after, the empirical inductive test $p_{I}^{\prime}$. This universality is not the product of empirical induction but a result of effective logical extension from a natural law to its corresponding quasi-law tautology. The logical extension is ensured by Theorem 1. Namely, the induction in empirical test does nothing to produce the universality but merely to carry the ready-made universality to a suitable recursive dimension. This explanation is quite different from the traditional understanding in the famous inductive problem. Physicists use a limited inductive sample to probabilistically test the universality of a physical law, which is ready-made in a quasi-law tautology. In recursive abduction, theoretically, quasi-law-tautologically based universality can be tested in limited steps in any suitable recursive dimension without the problem of infinite induction; and, practically, the ready-made universality can be tested by empirical induction in a limited inductive sample, in which the probability argument is always feasible. So the universality of natural law is probabilistically testable by recursive abduction. The difficulty of inductive problem has been overcome for it is just resulted from a natural law's universality producable from a probabilistic examination.

The above recursive feature in $L$-abduction interprets the acceptance or rejection of a physical law not from numerously piling up enumerated empirical inductive evidences but from several key pieces of evidence in crucial experiments or empirical observations in $L$-abduction. And the repeatability of observation in testing a physical law (for acceptance or rejection) can be interpreted in the above recursive sense as well.

Quasi-law tautology discriminates the strict and non-strict empirical sciences. Physics is the strictest empirical science because almost of all its laws have their own quasi-law tautologies, and thus, its empirical truth is determined by recursive abduction, just like geometry. Psychology, biology, and clinical medicine, and etc., all are sub-strict empirical sciences for some of their empirical truths come from recursive abduction and some from normal abduction. It is the recursive abduction to contribute the universality to physics and endow the particular connotation to the universality of physics. In recursive abduction, the universality of physics is verifiable and falsifiable recursively, just like a geometry used in physical space measurement. Recursive abduction gives a clear, smooth, and coherent non-monotonic and dynamic reasoning path.

We have clarified now that

1. For any candidate of natural law derived from a quasi-law tautology, the logical consistent explanation requests it to be recursive in all suitable recursive dimensions; and it is only a formal result in logic similar to a formal geometry.

2. When a formally required recursive candidate of natural law is tested in L-abduction experimentally or empirically, the test becomes realistic recursive abduction.

3. Only the quasi-law tautological systems, such as physics, geometries, and etc., can be examined in recursive abduction; classical logic and normal abductive systems cannot do it.

4. The empirical test to the universality of a quasi-law tautological system is essentially an empirical test in recursive abduction.

5. In recursive abduction, the universality of a strict natural law is not produced from empirical induction, and scientists just use limited inductive sample to probabilistically test the universality of a strict natural law, which is ready-made in a quasi-law tautology. So the probability argument for natural law is valid.

\section{Discussion on Hume's Problem}

In Hume's problem, the continuously besetting difficulty in history was that all universal natural laws were sweepingly regarded as the result of empirical induction mixed with common sense; however, any empirical 
induction was predestined to be limited and, thus, failed to offer a universal result. There is no effective logical path for creating a universal natural law purely from empirical induction. Now, from the $L$-abductive point of view, we have known that in Hume's problem one always misunderstood a formal universality derivable from quasi-law tautology as an empirical conformability about past to future and a time-recursive issue as an infinitely inductive problem and, hence, sank into an inescapable quagmire.

(3.3) and (3.4) presents an effective logical path for connecting a universal natural law with finite empirical observation, in which the universality of a natural law is derivable in (3.3) as a formal result similar to the geometrical theorem before it is examined empirically, and then, the empirical truth value is decidable in the recursive shcema (3.4) without infinite induction being needed. It provides a new foundation for the resolution of Hume's problem. This new logical framework clearly distinguishes universality and time recursion for a natural law and excludes the confusion commonly made by Hume and his opponents. The time recursion is a more basic feature independent from the contents of a natural law. They are not equivalently fundamental as thinking elements just like natural number in mathematics.

In a quasi-law tautology such as (3.1), letting $t_{k}, k=0,1,2, \cdots$, cover the past and future, we go into typical Hume's problem. Time recursion as the most fundamental attribute is basic for natural laws, Hume's critique, and the counter-proposal to Hume's critique, and it is thus the common ground on which they can engage in a battle. In other words, Hume's problem is also based itself on time recursion. When we discuss Hume's problem, it is enough to assume the concept of time recursion but we need not discuss time recursion itself. Or in short, if recursive abduction is first-order logic, natural law, Hume's critique, and the counter-proposal to Hume's critique all are second-order systems with respect to time.

Under the viewpoint of $L$-abduction, as discussed in detail above, the quasi-law tautology and its conclusion will keep make the past or the future irrelevant. This is requested by the logical consistent explanation in the quasi-law tautology, and is a formal requirement just like that in geometry. Hume thought that future truth is only possibly coming from past inductive one, and did not understand both the consistent demand from a quasi-law tautology and the empirical test in time-recursive abduction. In fact, the logical consistent explanation in a quasi-law tautology not only requests that the empirical truth of future must be coherent to the past but also requests that the past one must be coherent to the future. It is just the feature of any empirical science based on $L$-abduction. Hume's problem to the strict natural law system is a pseudo problem.

In Case 1, introduce the temporal characteristics to $\mathcal{L}_{G}$ as follows

$$
\begin{aligned}
& \left(\neg v_{0} \rightarrow \neg h_{\uparrow}\right) \rightarrow\left(e \rightarrow\left(h_{\uparrow} \rightarrow v_{0}\right)^{P}\right), \\
& \left(\neg v_{0} \rightarrow \neg h_{\uparrow}\right) \rightarrow\left(e \rightarrow\left(h_{\uparrow} \rightarrow v_{0}\right)^{F}\right),
\end{aligned}
$$

where, ()$^{P}$ and ()$^{F}$ indicate the sentences within ( ) belonging to the past and future, respectively.

Hume's critique is equivalent to say that one can only accept (4.1) and must reject (4.2). As we had discussed, (4.1) and (4.2) are equivalent in the time-recursive sense and the consistent explanations and conclusions of (4.1) and (4.2) keep the same to the original $\mathcal{L}_{G}$. It is emphasized that (4.1) and (4.2) equivalently and simultaneously hold the same as formula (2.2), by no means can (4.2) only be derived from (4.1). It is a request from the logical consistent explanation in (2.2) but not an additional empirical hypothesis regarding conformability in the past and future. Its empirical truth value is determined by $L$-abduction of time-recursive empirical test. Here, the key point is that $\mathcal{L}_{G}$ requests the same consistent explanation in the past and future among observables $h_{\downarrow}, h_{\uparrow}, v_{0}$, and $v_{0 \downarrow}$. This feature is quiet familiar to us in geometry when we use it to describe the realistic world. And this feature does not exist in any normal empirical inductive conclusion for which there is no any logically consistent explanation given in a quasi-law tautology. Hume and his opponents all overlooked the logical intercommunity represented by 
$\mathcal{L} p_{L}$ in the natural law and geometry, and completely mistook the emphasis on natural law.

We can further explain (4.1) and (4.2) by examples to eliminate possible divergence as follows: Newtonian mechanics as a theoretical system in its applicable field always keeps its own consistent explanation regardless of it being a precise empirical theory before the twentieth century or as an approximation after the twentieth century, just like Euclidean geometry always has had its own consistency regardless of the more precise empirical observation accepting or rejecting it. The consistency requires all observables to be consistently related to each other whether past or future. Hume's skepticism only questions the normal empirical inductive process but does not apply to a quasi-law-tautologically based natural law. The twentieth-century negative evidence regarding Newtonian mechanics changes the implication of its always positive evidence in the seventeenth century, which in fact can be explained as a good approximation to the twentieth-century evidence for low-speed and macroscopic phenomena; and vice versa, the seventeenth-century positive evidence can ask the twentieth-century evidence to consistently agree with it. This is not the attribute of a normal inductive result. For a normal inductive conclusion such as "Tom always dresses in red colors", if we saw Tom dressed in green colors today, we would regard the inductive conclusion wrong today but unnecessarily had to change the meanings of past evidence "Tom dressed in red colors yesterday"; and vice versa, "Tom dressed in red colors yesterday" does nothing to the question consistently asked "Would Tom dress in red colors in the future?" To the normal empirical inductive result, Hume's question is proper. The difference between quasi-law-tautologically based natural laws and normal empirical inductive conclusions can be illustrated as follows: Newtonian mechanics has consistently provided explanations, excluding contradictory evidence, regardless of past and future, but a normal empirical inductive conclusion only contains the simple accumulation of observable evidence un-exclusive to the contradiction, without any consistent asking to its contents in any dimension included in the past-future dimension and, therefore, is suitable to be questioned by Hume's skepticism. It is all too obvious that any empirical support to a consistent theory is logically indifference to the past and future for its logical consistent explanation. It is a logical attribute regardless of time. Empirical observables as the contents in a physical law are always organized together consistently to make the whole so that the future (or past) negative evidence will be invalid unless it meanwhile consistently changes the past (or future) one and includes the past (or future) one. The future suitability of a physical law merely refers to nothing but the consistent restriction for all observables irrelevant to past or future, if time is a suitable recursive dimension. Facing the empirical test, a normal empirical inductive conclusion is only a weak and fragile defendant, but a strict natural law has the strong consistence power to persist in its own "ask" for accepting or rejecting it. By the way, not involving rigorous logical contents and mainly focusing on the factual outcome, Lakatos [51] from a historical and philosophical sight, to some extent, had similarly discussed the same result called "hard core" and "protection zone" of scientific theory. In this paper, the conclusion is more clear and explicit logically. In this way, a natural law is always universally applicable to past and future as well as it is universally falsifiable in any time. It is the logical consistent explanation in a quasi-law tautology to request but not to examine the physical law applicable from past to future in recursive time dimension. And this attribute of a physical law completely resembles to the consistence between theorems and their axioms in a geometry which need not have to additionally suppose that in a natural law the contents of future must be conformable to the past.

In recursive $L$-abduction, any strict natural law must be consistent in past and future, and simultaneously to change its meanings in past and future if any counter evidence occurs. Namely, the past implies the future, meanwhile, the future implies the past, they are originally equivalent in recursive $L$-abduction; and the future never implies a one-way support from the past. This is the sense of the universality of natural law. Hume and his opponents all mistook the universality as the inductive result of one-way support from past to future.

This analysis reveals the key role of quasi-law tautologies in a physical law system. It is the quasi-law tautology to distinguish a physical law from the normal empirical commonsense and to have physical law immunized to the 
infection of Hume's skepticism.

Under the interpretation from $L$-abduction, the function of empirical induction $p_{I}^{\prime}$ in a scientific theory is to anchor logical consistent explanation in empirical phenomena by recursive abduction, but not to provide an enormous factual aggregation to pile up a natural law. The minimal observational set of $p_{I}^{\prime}$ is $\left\{p_{I}^{\prime r_{0}}, p_{I}^{\prime r_{k}}, p_{I}^{\prime r_{k+1}}\right\}$. In the recursive abduction, effective empirical anchoring asks for clear logical contexts but not the huge quantities of evidence.

Of course, observational repeatability is one of the important derived features of recursive abduction. Researchers always unconsciously use repeatability as a practically equivalent substitute for recursion to promote the efficiency of scientific discovery and, meanwhile, it absorbs researchers' attention to deviate from the essential character of recursive abduction. Numerous empirical evidences are in favor of the discovery of natural law for two reasons: first, people easily makes mistakes with merely one set of observations and repeatability is helpful to rule out one's carelessness; and second, a result derived from recursive $L$-abduction must contain repeatability in all recursive cases, hence, it will be more possible to find a recursive natural law in repeatable observations than in repeatability-unknown cases. In the first reason, the repeated inductive observation is a behavioral management measure to overcome the human being's behavioral fault; and in the second reason, the repeated inductive observation will reduce the discovery cost in scientific activities, is a cost-saving principle. In current discussions about Hume's problem, all participants were often at sea for they misunderstood a behavioral management measure and a cost-saving principle as the primitive attribute of natural law.

The discovery of scientific theory is a process to look for consistent explanation among empirical phenomena, just like geometry being used in the measurement of physical space. The logical consistent explanation given in $\mathcal{L} p_{L}$ requests all elements to be non-contradictory from each other in all suitable recursive dimensions including, but not limited to, the past and future. Hume's analysis only suits the critique of inductive commonsense, in which however originally no rigorous logical consistent explanation is sought, and to the immature sub-science, such as the penal data model in economics, traditional folk medical diagnosis, and etc., which are however soft and unreliable data. Hume's problem is hence not only inapplicable to strict natural laws but also redundant, though is not false, as a rational critique to empirical induction or commonsense.

\section{Conclusions}

It is concluded from this paper:

1. Case studies reveal the quasi-law tautology functioning in the derivation for any physical law. Quasi-law tautology combines with abduction naturally to lead to recursive abduction.

2. The empirical universality of physical law is based on recursive abduction. It refers to nothing else but a set of consistent physical relations holding in one or more suitable recursive dimensions, just like a consistent geometry used in the measurement of realistic physical world. In the schema of recursive abduction, a physical law is always derivable logically and decidable empirically.

3. Hume's problem and its typical opponent proposals all misunderstood a formal universality derivable from quasi-law tautology as an empirical conformability about past to future and a time-recursive issue as an infinitely inductive problem and, thus, sank into an inescapable quagmire. From the viewpoint of recursive abduction Hume's problem naturally vanishes.

4. Especially, recursive abduction provides a new logical framework to accommodate the probability argument for the universality of natural laws. In this new framework, the calculation of a confirmation probability is based on a limited empirical inductive sample and it is always empirically feasible without the problem of zero probability in an infinitely inductive sample. 


\section{References}

1. Pierce, C. S. On the Natural Classification of Arguments. 1867. Proceedings of the American Academy of Arts and Sciences, Vol. 7 (May, 1865 -May, 1868), 261-287.

2. Meheus, J. and Batens, D. A Formal Logic for Abductive Reasoning. Logic Journal of IGPL, 2006, 14(2), 221-236.

3. Lycke, H. A Formal Explication of the Search for Explanations: The Adaptive Logics Approach to Abductive Reasoning. Logic Journal of IGPL, 2012, 20 (2), 497-516.

4. Soler-Toscano, F., Fernndez-Duque, D., and Nepomuceno-Fernndez, N. A Modal Framework for Modelling Abductive Reasoning. Logic Journal of IGPL, 2012 , 2 (2), 438-444.

5. Beirlaen, M. and Aliseda, A. A Conditional Logic for Abduction. Synthese, 2014, 191(15), 3733-3758.

6. Nubiola, J. Abduction or the Logic of Surprise. Semiotica, 2005, 153(1),117-130.

7. Pombo, O. and Gerner, A. Abduction and the Process of Scientific Discovery. Lisbon: CFCUL/Publidisa, 2007.

8. McNally, R. J., Lasko, N. B., Clancy, S. A., Macklin, M. L., Roger K. Pitman, R. K., and Orr, S. P. Psychophysiological Responding during Script-Driven Imagery in People Reporting Abduction by Space Aliens. Psychological Science, 2004, 15(7), 493-497.

9. Chattopadhyay, I. and Lipson, H. Abductive Learning of Quantized Stochastic Processes with Probabilistic Finite Automata. Philosophical Transactions: Mathematical, Physical and Engineering Sciences, Signal Processing and Inference for the Physical Sciences. 2013, 371, 1-22.

10. Haig, B. D. Detecting Psychological Phenomena: Taking Bottom-Up Research Seriously. The American Journal of Psychology, 2013, 126(2), 135-153.

11. Khemlani, S. S., Mackiewicz, R., Bucciarelli, M., and Johnson-Laird, P. N. Kinematic Mental Simulations in Abduction and Deduction. Proceedings of the National Academy of Sciences of the United States of America, 2013, 110(42), 16766-16771.

12. Pedemonte, B. and Reid, D. The Role of Abduction in Proving Processes. Educational Studies in Mathematics, 2011, 76(3), 281-303.

13. Bajc, V. Abductive Ethnography of Practice in Highly Uncertain Conditions. The Annals of the American Academy of Political and Social Science: Contemporary Urban Ethnographic Research. 2012, 642, 72-85.

14. Singer, B. Comment: Implication Analysis as Abductive Inference. Sociological Methodology, 2008, 38, 75-83.

15. Hume, D. The Treaties of Human Nature. 1739. London and Glasgow: Collins, 1962.

16. Hume, D. Enquiry into the Human Understanding. 1748. Oxford: Clarendon Press, 1963.

17. Fisher, P. A. The Design of Experiments. London: Oliver and Boyd, 1935

18. Carnap, R. On the Application of Inductive Logic. Philosophy and Phenomenological Research, 1947, 8 (1), 133-148.

19. Carnap, R. Logical Foundations of Probability. Chicago: University of Chicago Press, 1950.

20. Carnap, R. The Continuum of Inductive Method. Chicago: University of Chicago Press, 1952.

21. Carnap, R. A Basic System of Inductive Logic. In R. Carnap and R. C. Jeffrey (Eds.), Studies in Inductive Logic and Probability. Berkeley: University of California Press, 1971. 33-167.

22. Reichenbach, H. The Theory of Probability ( $2^{\text {nd }}$ Ed). Berkeley and Los Angeles: University of California Press, 1949.

23. Reichenbach, H. The Rise of Scientific Philosophy. Berkeley and Los Angeles: University of California Press, 1954. 
24. Worrall, J. Structural Realism: The Best of Both Worlds. In D. Papineau (Eds.), Philosophy of Science. Oxford: Oxford University Press, 1996.

25. Kelly, K. The Logic of Reliable Enquiry. Oxford: Oxford University Press, 1996.

26. Popper, K. R. The Logic of Scientific Discovery. London and New York: Hutchinson \& Co, 1959.

27. Popper, K. R. Objective Knowledge. Oxford: Oxford University Press, 1970.

28. Popper, K. R. Realism and the Aim of Science. London: Rowman and Littlefield, 1983.

29. Popper, K. R. Why Probabilistic Support is not Inductive. Philosophical Translations of the Royal Society of London, 1987, (A321), 568-591.

30. Shier, J. The Unsolvability of Hume's Problem and the Local Justification of Induction. Epistemologia, 1993, XVI, 77-96.

31. Howson, C. Hume's Problem: Induction and the Justification of Belief. Oxford: Clarendon Press, 2000.

32. Skyrms, B. Pragmatics and Empiricism. New Haven and New York: Yale University Press, 1984, 51-68.

33. Boulter, S. J. Hume on Induction: A Genuine Problem or Theology's Trojan Horse? Philosophy, 2002, 77( 299), 67-86.

34. Okasha, S. Does Hume's Argument against Induction Rest on a Quantifier-Shift Fallacy? Proceedings of the Aristotelian Society, New Series, 2005,105, 237-255

35. Hetherington, S. Not Actually Hume's Problem: On Induction and Knowing-How. Philosophy, 2008, 83(326), 459-481.

36. Schurz, G. The Meta-inductivist's Winning Strategy in the Prediction Game: A New Approach to Hume's Problem. Philosophy of Science, 2008, 75(3), 278-305.

37. Goodman, N. A Query on Confirmation Theory. Journal of Philosophy, 1946, 43 (14), 383-385.

38. Goodman, N. Fact, Fiction, and Forecast. Cambridge: Harvard University Press, 1955, 63-83.

39. Barker S. F. and Achinstein, P. On the New Riddle of Induction. Philosophical Review, 1960, 69 (4), $511-522$.

40. Small, K. Professor Goodman's Puzzle. Philosophical Review, 1961, 70 (4), 544-552.

41. Ullion, S. More on "Grue" and Grue. Philosophical Review, 1961, 70 (3), 386-389.

42. Quine, W. V. O. Epistemology Naturalized, Ontological Relativity and Other Essays. New York and London: Columbia University Press, 1969, 69-90

43. Hesse, M. The Structure of Scientific Inference. Oakland: University of California Press, 1974.

44. Israsel, R. Two Interpretation of "Grue" - or How to Misunderstand the New Riddle of Induction. Analysis, 2004, 64 (4), 335-339.

45. Chart, D. Schulte and Goodman's Riddle. The British Journal for the Philosophy of Science, 2000, 51(1), 147-149

46. Godfrey-Smith, P. Goodman's Problem and Scientific Methodology. The Journal of Philosophy, 2003, 100(11), 573-590.

47. Schwartz, R. A Note on Goodman's Problem. The Journal of Philosophy, 2005, 102(7), 375-379.

48. Galilei, G. Dialogue Concerning Two New Sciences, 1638. H. Crew and A. Salvio (Tran.), Macmillan Publishers, 1914, 244-279.

49. Aristotle, Physics, B.C. R. P. Hardie, and R. K. Gaye, (Trans.) eBooks@Adelaide, https://ebooks. adelaide.edu.au/a/aristotle/physics/. Book VIII, 2015.

50. Kuhn, T. S. (1962). The Structure of Scientific Revolution ( $2^{\text {nd }}$ ed). Chicago: University of Chicago Press. 26-30.

51. Lakatos, I. (1976). The Methodology of Scientific Research Programmes. London: Cambridge University Press. Chapter 1. 